\title{
The combined use of miRNAs and mRNAs as biomarkers for the diagnosis of papillary thyroid carcinoma
}

\author{
YINLONG ZHAO $^{1,2}$, XIAODONG LIU ${ }^{1}$, LILI ZHONG ${ }^{3}$, MENGZI HE ${ }^{1}$, \\ SILIN CHEN ${ }^{1}$, TIEJUN WANG ${ }^{4}$ and SHUMEI MA ${ }^{1,5}$
}

Received March 25, 2015; Accepted July 29, 2015

DOI: $10.3892 / \mathrm{ijmm} .2015 .2305$

\begin{abstract}
Thyroid carcinoma (TC) is the most common malignancy of the endocrine system, and papillary thyroid carcinoma (PTC) accounts for the largest proportion of cases with TC. Although histology is considered the gold standard in the diagnosis of PTC, the sensitivity and specificity of this method is low. Therefore, developing novel diagnostic and prognostic biomarkers for PTC is essential. MicroRNAs (miRNAs or miRs) and their target RNAs play critical roles in tumorigenesis and tumor progression. Thus, the characteristic miRNA and mRNA expression profiles may function as diagnostic biomarkers for tumors, making it possible to predict the tumor stage and the prognosis of patients. In the present study, we detected miRNAs and mRNAs which can function as novel biomarkers for the diagnosis of PTC. The sensitivity of the diagnostic tests was evaluated by receiver operating characteristic curve analysis. Pearson's correlation analysis was used to determine the correlation between mRNAs and miRNAs, and cancer types. We found that the area under the curve (AUC) values of 8 miRNAs (miR-106a, miR-15a, miR-30a, miR-30b, miR-20a, miR-20b, miR-30d and miR-30e) and 8 mRNAs [axis inhibition protein 2 (AXIN2), integrin, alpha 3 (antigen CD49C, alpha 3 subunit of VLA-3 receptor) (ITGA3), tumor protein p53 inducible nuclear protein (TP53INP)1, TP53INP2, B-cell CLL/lymphoma 2 (BCL2), phosphatase and tensin homolog (PTEN), FOS and K(lysine) acetyltrans-
\end{abstract}

Correspondence to: Dr Shumei Ma, Key Laboratory of Radiobiology (Ministry of Health), School of Public Health, Jilin University, 1163 Xinmin Street, Changchun, Jilin 130021, P.R. China

E-mail: shmm2001@126.com

Dr Tiejun Wang, Department of Radiation Oncology, The Second Hospital of Jilin University, 218 Ziqiang Street, Changchun, Jilin 130021, P.R. China

E-mail: m13943016598@163.com

Key words: papillary thyroid carcinoma, biomarker, miRNA, mRNA ferase $2 \mathrm{~B}$ (KAT2B)] were $>0.90$. The combination of miR-15a and AXIN2 significantly improved the diagnostic accuracy. Therefore, our data indicate that the differential expression of miRNAs combined with that of their target mRNAs may serve as a powerful biomarker for distinguishing PTC from benign tissues.

\section{Introduction}

Thyroid carcinoma (TC) is the most common malignancy of the endocrine system. An estimated 62,980 new cases of TC were diagnosed, and approximately 1,890 deaths were caused by TC in the United States in 2014 (1). TC is commonly diagnosed at a younger age than the majority of other adult cancers (2). The 4 main histological types of TC are papillary thyroid carcinoma (PTC), follicular thyroid carcinoma (FTC), medullary thyroid carcinoma (MTC) and anaplastic (undifferentiated) carcinoma (ATC) $(3,4)$. PTC and FTC constitute approximately $90 \%$ of total number of TC cases and are treatable and usually curable. However, both PTCs and FTCs may progress to poorly differentiated thyroid carcinomas (PDTCs) or may completely lose differentiation and transform into ATC, a type of poorly differentiated TC, which is aggressive, prone to early metastasis and is associated with a poor prognosis (5). Histology is considered the gold standard for TC diagnosis. However, it is difficult to distinguish between PTC and FTC under a microscope (6). Furthermore, conventional histology fails to provide prognostic and therapeutic information for TC. Some biomarkers, such as thyroglobulin $(\mathrm{Tg})(7,8)$, galectin-3 (9) and HBME-1 (10), have been used in clinical practice for the diagnosis of PTC; however, the sensitivity and specificity of these biomarkers are low, and only a small fraction of these biomarkers can be used as diagnostic or prognostic biomarkers. Therefore, it is essential to develop novel diagnostic and prognostic biomarkers for PTC.

MicroRNAs (miRNAs or miRs), a class of short non-coding RNAs with a length of 19-22 nucleotides, and play important roles in tumorigenesis and cancer progression $(11,12)$. miRNAs regulate gene expression at the post-transcriptional 


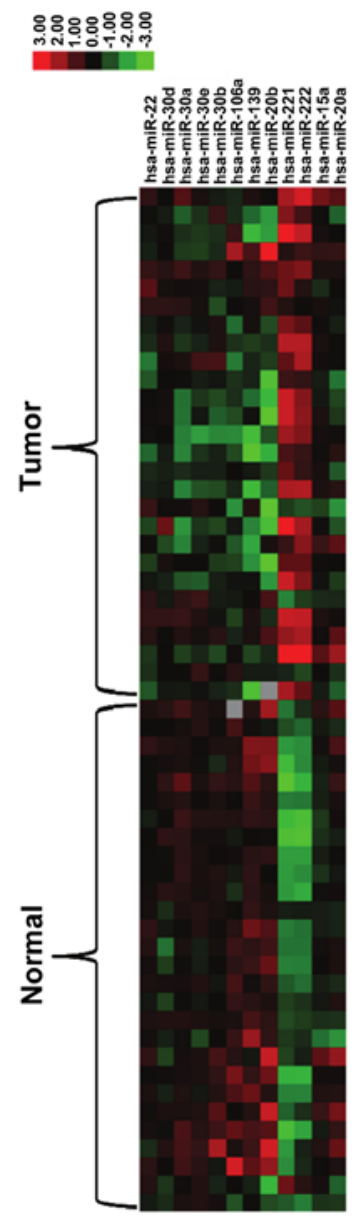

Figure 1. MicroRNA (miRNA) expression analysis using the data of 28 patients with papillary thyroid carcinoma (PTC) obtained from The Cancer Genome Atlas (TCGA). Color gram of miRNA expression profiles of patients with PTC. All miRNAs were divided into 2 groups: upregulated (red) and downregulated (green).

level by binding to the 3'-UTR of their target mRNAs (13). A number of studies have demonstrated that miRNA expression is associated with cell proliferation, metastasis, invasion and and response to therapy (14-20). miRNA expression differs between cancer tissues and adjacent normal tissues in patients (21-23). These data indicate that miRNAs may be used as potential biomarkers for the diagnosis and prognosis of patients with cancer.

In the present study, we examined the expression profiles of miRNAs and mRNAs in patients with PTC and evaluated their potential for use as biomarkers for PTC diagnosis. The differential expression of miRNAs, combined with that of their target mRNAs, may serve as a powerful biomarker for distinguishing PTC from benign tissues.

\section{Materials and methods}

Datasources.miRNA expression data,transcription sequencing (RNA-Seq) data and the corresponding clinical information for 28 patients with PTC were obtained from The Cancer Genome Atlas (TCGA) data portal (http://cancergenome.nih. gov). This database is freely available for non-commercial and academic use. The TCGA data, as well as the cBioPortal for Cancer Genomics (http://www.cbioportal.org) and Oncomine

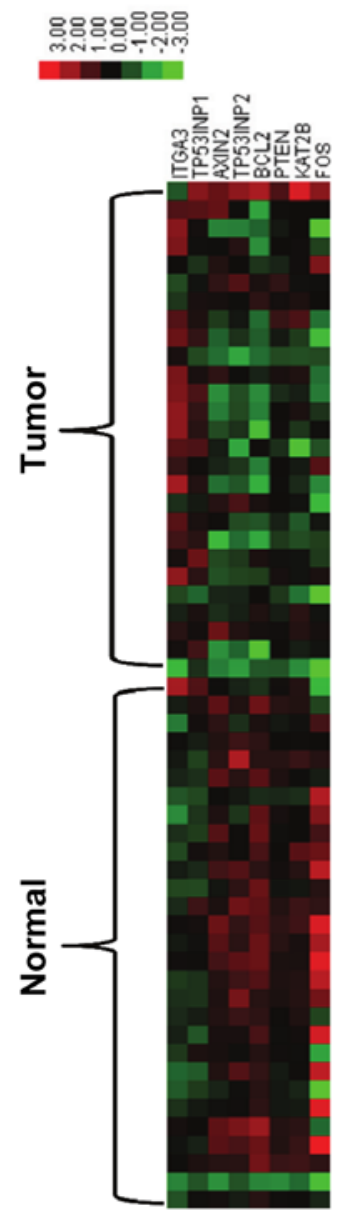

Figure 2. Gene expression analysis using the data of 28 patients with papillary thyroid carcinoma (PTC) obtained from The Cancer Genome Atlas (TCGA) Color gram of mRNA expression profiles of patients with PTC. All mRNAs were divided into 2 groups: upregulated (red) and downregulated (green).

(http://www.oncomine.org) data were in the form of RNA sequencing data on an array platform. The sequencing data from TCGA were available in the form of 'reads per million (level 3)' for each miRNA. As regards RNA-Seq gene expression, only data from patients with matched tumor and normal samples were used. cBioPortal and Oncomine were also used to examine the expression of miRNAs and RNAs from the TCGA data portal. miRNA expression analysis of the 28 patients with PTC in the TCGA data portal was carried out using the software package TreeView version 1.1.

Receiver operating characteristic (ROC) curve analysis. To evaluate the sensitivity of the diagnostic tests, ROC curve analyses were performed using MedCalc ${ }^{\circledR}$ statistical software (11.4.2.0; MedCalc statistical software, Mariakerke, Belgium). The area under the ROC curve (AUC), which has been described as a simple and convenient overall measure of diagnostic test accuracy, represents the probability and correspondence between the ROC curve and the tested factors.

miR-15a/axis inhibition protein 2 (AXIN2) expression in other types of cancer. Pearson's correlation analysis was used to determine the correlation between mRNAs and miRNAs, and cancer types, including $\mathrm{p}$-values and the false discovery rate (FDR). 

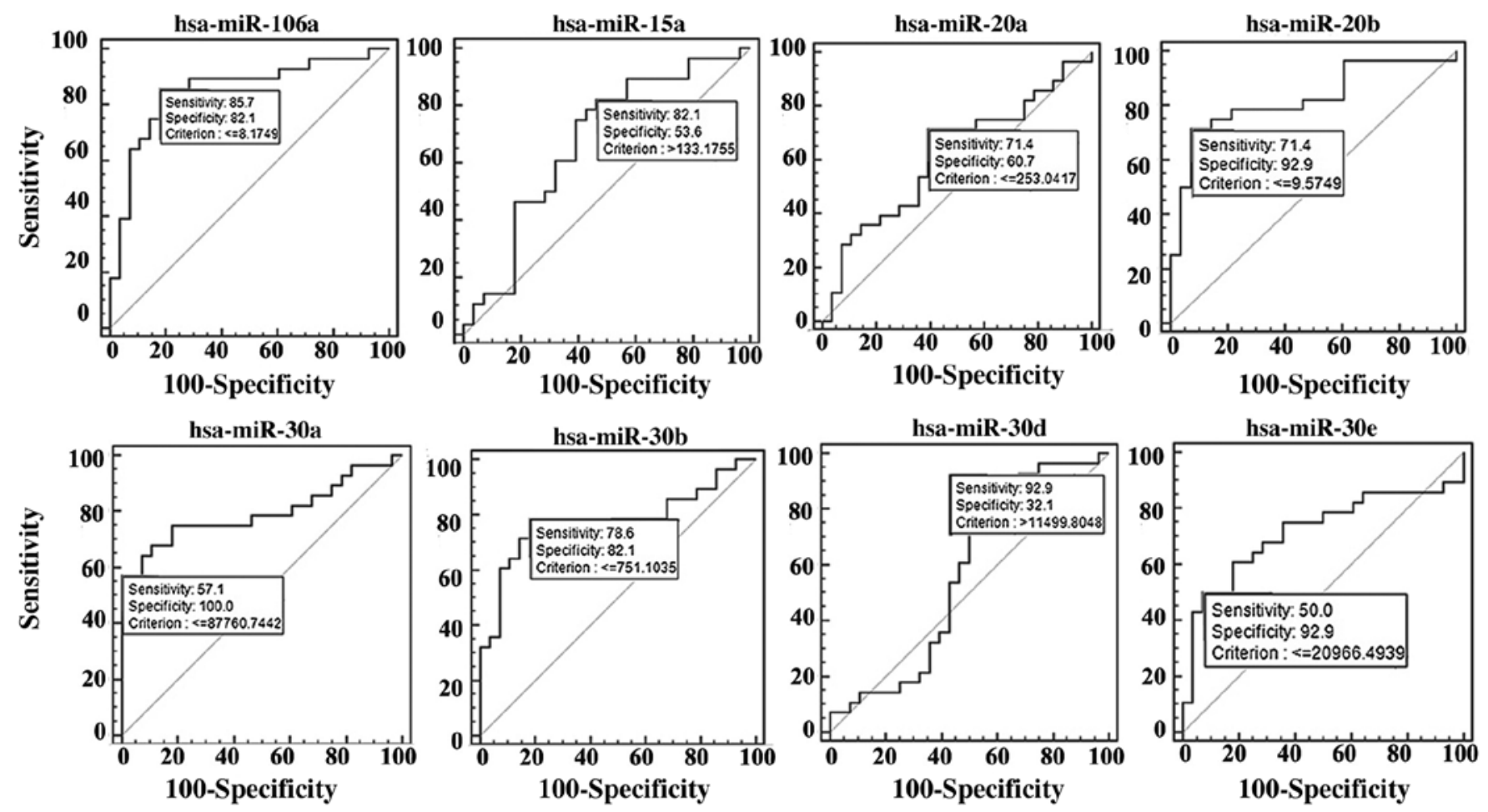

Figure 3. Receiver operating characteristic (ROC) curves of the 8-miRNA signature in patients with papillary thyroid carcinoma (PTC).ROC curve analysis of miR-106a, miR-15a, miR-20a, miR-20b, miR-30a, miR-30b, miR-30d and miR-30e in tumors and normal tissues.

Statistical analysis. All statistical analyses were carried out using SPSS for Windows, version 19.0 (SPSS, Inc., Chicago, IL, USA). Combined predictors were established using the logistic regression method. ROC curves were established to evaluate the diagnostic effects of miRNAs. The results are expressed as the means $\pm \mathrm{SD}$. P-values $<0.05$ were considered to indicate statistically significant differences.

\section{Results}

Screening of differentially expressed miRNAs. Both tumor tissues and matched normal tissues from the same patient were used for miRNA expression profile analysis. The data of 28 patients with PTC, from TCGA, were included in the present study. miRNA expression was calculated from 'reads per million' values of the tumor and matched normal samples. We found that 12 miRNAs (miR-20a, miR-15a, miR222, miR-221, miR-20b, miR-139, miR-106a, miR-30b, miR-30e, miR-30a, miR-30d and miR-22) demonstrated a $>2$-fold difference in expression between the tumor tissues and normal tissues in $70 \%$ of the patients. The upregulated and downregulated miRNAs are presented in Fig. 1.

Screening of differentially expressed genes. We further examined differentially expressed genes in the tumor tissues and matched normal tissues in the 28 patients with PTC. A total of 8 genes [integrin, alpha 3 (antigen CD49C, alpha 3 subunit of VLA-3 receptor) (ITGA3), tumor protein p53 inducible nuclear protein (TP53INP)1, AXIN2, TP53INP2, B-cell CLL/lymphoma 2 (BCL2), phosphatase and tensin homolog (PTEN), K(lysine) acetyltransferase 2B (KAT2B) and FOS] were identified as differentially expressed between the PTC tissues and the matched normal thyroid tissues. The upregulated and downregulated genes are presented in Fig. 2.

ROC curve analysis of the differentially expressed miRNAs. The differentially expressed miRNAs in the PTC tissue samples were selected for further analysis. ROC curve analysis was performed on 28 tumor and 28 normal tissues to determine whether these miRNAs are related to the PTC histological status. The miRNAs, miR-106a, miR-15a, miR-20a, miR-20b, miR-30a, miR-30b, miR-30d and miR-30e, were found to be associated with PTC (Fig. 3). All of their AUC values were $>0.90$, and thus, this indicates that these miRNAs can be used as effective biomarkers for the diagnosis of PTC.

ROC curve analysis of the differentially expressed genes. ROC curve analysis was then carried out on the basis of the results from obtained using the PTC tissues, as compared with those obtained using the normal tissues. The expression of the target genes, AXIN2, ITGA3, TP53INP1, TP53INP2, BCL2, PTEN, FOS and KAT2N, was found to be associated with PTC (Fig. 4). All of these genes exhibited high sensitivity $(60.7,71.4,64.3,82.1,89.3,85.7,89.3$ and $85.7 \%$, respectively) and specificity $(92.9,96.4,85.7,75.0,92.9,46.4$, 63.9 and $67.3 \%$, respectively).

The potential value of combined biomarkers. We further examined the potential for using miRNAs combined with their target mRNAs in the diagnosis of PTC. ROC curve analysis revealed that when miR-15a was combined with its 

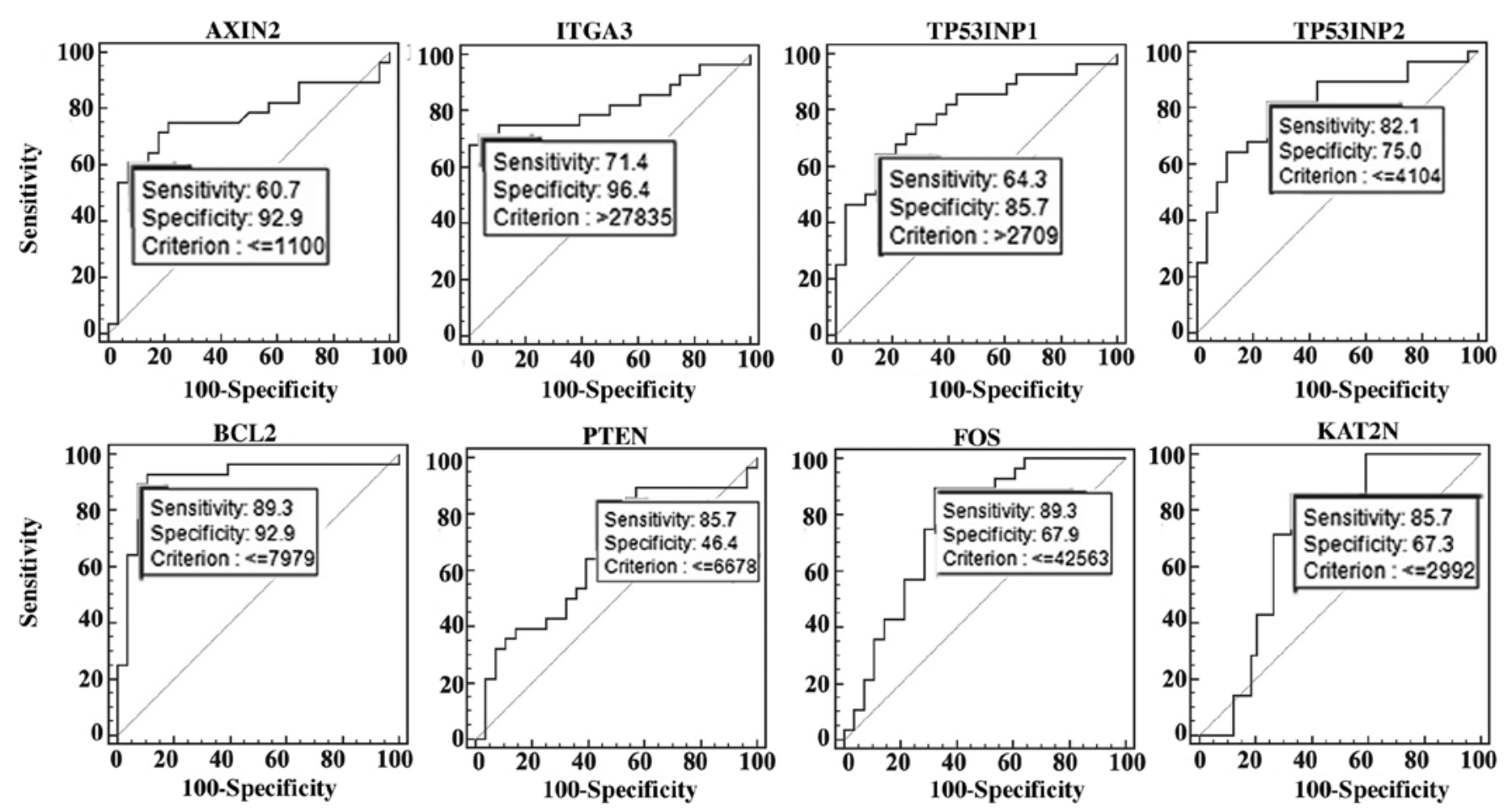

Figure 4. Receiver operating characteristic (ROC) curves of the 8-mRNA signature in patients with papillary thyroid carcinoma (PTC). ROC curve analysis of AXIN2, ITGA3, TP53INP1, TP53INP2, BCL2, PTEN, FOS and KAT2N in tumors and normal tissues.

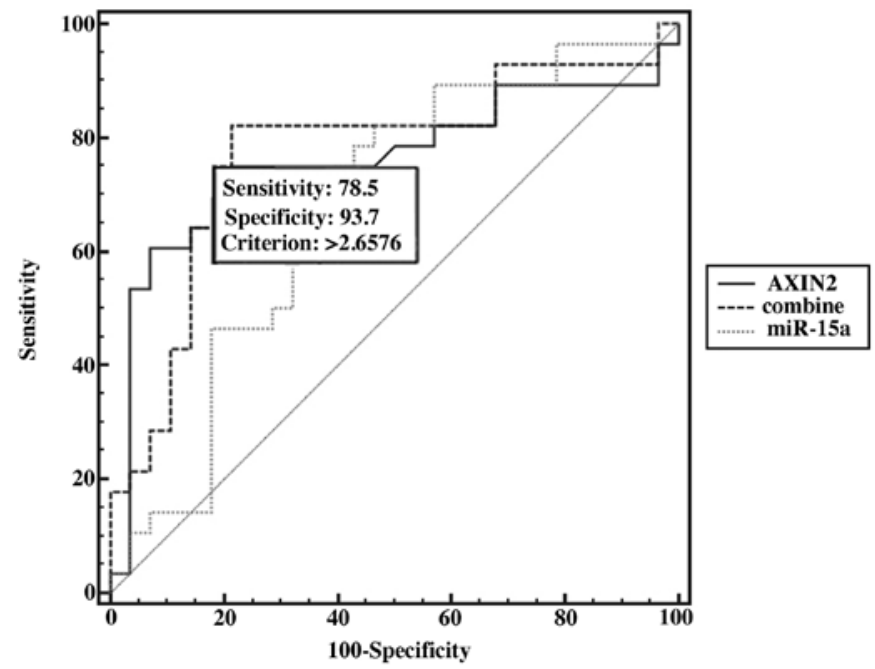

Figure 5. Receiver operating characteristic (ROC) curves of miR-15a combined with AXIN2 as a biomarker for the diagnosis of patients with papillary thyroid carcinoma (PTC).

target gene, AXIN2, the AUC values increased, and miR-15a combined with AXIN2 improved the sensitivity (78.5\%) and specificity (93.7\%) (Fig. 5). Moreover, we found that miR-15a and AXIN2 expression were changed coordinately in 8 types of cancer, as shown in Table I. We also analyzed the expression of the other miRNAs and their target genes in different types of cancer (Table I). Our results suggest that these miRNAs and mRNAs may be used as potential biomarkers for the diagnosis of PTC.

\section{Discussion}

The current clinical approaches for the diagnosis of PTC include researching patient history, physical examination, imaging, fine-needle aspiration (FNA) and surgical pathology. FNA and surgical pathology are the gold standard for the diagnosis of PTC. However, both methods are invasive and their predictive value is limited. Therefore, as has been described in a previous study, it is essential to identify novel biomarkers to predict the diagnosis and prognosis of patients with PTC (24). Previous studies have reported that certain miRNAs may be used as biomarkers for the diagnosis and prognosis of breast cancer and various diseases $(25,26)$. As compared with conventional protein-based biomarkers, certain miRNAs have several potential advantages, including easy detection by PCR, relative homogeneity and highly specific expression profiles (25).

Several analyses of miRNA and mRNA expression profiles have demonstrated that the study of the differential expression of miRNAs and mRNAs has potential value for tumor diagnosis and prognosis in patients with TC (27-30). Recent studies have demonstrated that some miRNAs have the potential to be used as diagnostic or prognostic markers for PTC $(24,31)$. Combining two or three markers constitutes a more accurate approach to differentiating malignant tumors from their benign counterparts when compared with using a single biomarker (32-34). However, to the best of our knowledge, no studies to date have examined the combined use of miRNAs and mRNAs as biomarkers for the diagnosis of TC. Previous studies have reported a series of differentially expressedmiRNAs and mRNAs in PTC (35-37). The differ- 
Table I. Expression of miRNAs and their target genes in different types of cancer.

\begin{tabular}{|c|c|c|c|c|c|c|c|}
\hline miRNA & $\begin{array}{l}\text { Target } \\
\text { gene }\end{array}$ & Cancer type & $\begin{array}{l}\text { Sample } \\
\text { no. }\end{array}$ & $\mathrm{r}$ & Rank & P-value & FDR \\
\hline \multirow[t]{8}{*}{ hsa-miR-15a } & \multirow[t]{8}{*}{ AXIN2 } & Bladder urothelial cancer (BLCA) & 229 & -0.29099 & 32222 & 7.58506E-06 & $9.23922 \mathrm{E}-05$ \\
\hline & & Breast cancer (BRCA) & 748 & -0.21574 & 53605 & $2.50939 \mathrm{E}-09$ & $1.84287 \mathrm{E}-08$ \\
\hline & & $\begin{array}{l}\text { Head and neck squamous cell } \\
\text { carcinoma (HNSC) }\end{array}$ & 428 & -0.34684 & 7364 & $1.52465 \mathrm{E}-13$ & $8.20853 \mathrm{E}-12$ \\
\hline & & Kidney renal clear cell carcinoma (KIRC) & 300 & -0.44051 & 9438 & $1.13431 \mathrm{E}-15$ & $4.65868 \mathrm{E}-14$ \\
\hline & & Lung adenocarcinoma (LUAD) & 441 & -0.12922 & 102516 & 0.00657988 & 0.0251174 \\
\hline & & Lung squamous cell carcinoma (LUSC) & 362 & -0.16374 & 101085 & 0.0017737 & 0.00692297 \\
\hline & & Papillary thyroid carcinoma (PTC) & 557 & -0.10371 & 164644 & 0.0143314 & 0.0344256 \\
\hline & & $\begin{array}{l}\text { Uterine corpus endometrial } \\
\text { carcinoma (UCEC) }\end{array}$ & 161 & -0.22901 & 59904 & 0.0034772 & 0.0227855 \\
\hline \multirow[t]{4}{*}{ hsa-miR-20b } & \multirow[t]{4}{*}{ TP53INP1 } & Kidney chromophobe $(\mathrm{KICH})$ & 91 & -0.37705 & 57043 & 0.000229526 & 0.00156243 \\
\hline & & Kidney renal clear cell carcinoma (KIRC) & 300 & -0.23217 & 71929 & 4.90559E-05 & 0.000264361 \\
\hline & & Lung adenocarcinoma (LUAD) & 441 & -0.11523 & 121627 & 0.015477 & 0.0497973 \\
\hline & & Papillary thyroid carcinoma (PTC) & 557 & -0.19305 & 69177 & 4.4499E-06 & $2.54406 \mathrm{E}-05$ \\
\hline \multirow[t]{6}{*}{ hsa-miR-106a } & \multirow[t]{6}{*}{ TP53INP1 } & Breast cancer (BRCA) & 748 & -0.13769 & 120249 & 0.000158461 & 0.000518767 \\
\hline & & Colorectal cancer (CRC) & 299 & -0.33271 & 9916 & 3.68032E-09 & $1.4467 \mathrm{E}-07$ \\
\hline & & Kidney chromophobe $(\mathrm{KICH})$ & 91 & -0.36623 & 61281 & 0.000356682 & 0.00226009 \\
\hline & & Kidney renal clear cell carcinoma (KIRC) & 300 & -0.22882 & 73995 & 0.000063344 & 0.000331829 \\
\hline & & Lung squamous cell carcinoma (LUSC) & 362 & -0.19645 & 74972 & 0.000168949 & 0.00088911 \\
\hline & & Papillary thyroid carcinoma (PTC) & 557 & -0.13739 & 120320 & 0.00115165 & 0.00378548 \\
\hline \multirow[t]{10}{*}{ hsa-miR-20a } & \multirow[t]{10}{*}{ TP53INP2 } & Bladder urothelial cancer (BLCA) & 229 & -0.49808 & 2457 & $9.24247 \mathrm{E}-16$ & $1.47643 \mathrm{E}-13$ \\
\hline & & Breast cancer (BRCA) & 748 & -0.33902 & 11844 & $1.42082 \mathrm{E}-21$ & $4.7225 \mathrm{E}-20$ \\
\hline & & Colorectal cancer (CRC) & 299 & -0.17681 & 63670 & 0.00214911 & 0.0131569 \\
\hline & & $\begin{array}{l}\text { Head and neck squamous } \\
\text { cell carcinoma (HNSC) }\end{array}$ & 428 & -0.26834 & 24475 & 1.71039E-08 & $2.77065 \mathrm{E}-07$ \\
\hline & & Kidney chromophobe $(\mathrm{KICH})$ & 91 & -0.32027 & 82252 & 0.00196898 & 0.00929535 \\
\hline & & Kidney renal clear cell carcinoma (KIRC) & 300 & -0.17722 & 113053 & 0.00206243 & 0.00707144 \\
\hline & & Lung squamous cell carcinoma (LUSC) & 362 & -0.31744 & 23413 & $6.43229 \mathrm{E}-10$ & $1.08395 \mathrm{E}-08$ \\
\hline & & Skin cutaneous melanoma (SKCM) & 342 & -0.15318 & 71854 & 0.00452332 & 0.0247739 \\
\hline & & Papillary thyroid carcinoma (PTC) & 557 & -0.14565 & 111172 & 0.000564255 & 0.00200733 \\
\hline & & $\begin{array}{l}\text { Uterine corpus endometrial } \\
\text { carcinoma (UCEC) }\end{array}$ & 161 & -0.3447 & 18090 & 7.54732E-06 & 0.000163772 \\
\hline \multirow[t]{7}{*}{ hsa-miR-15a } & \multirow[t]{7}{*}{ BCL2 } & Bladder urothelial cancer (BLCA) & 229 & -0.40527 & 8501 & $1.83006 \mathrm{E}-10$ & 8.44936E-09 \\
\hline & & Breast cancer (BRCA) & 748 & -0.21583 & 53550 & 2.46948E-09 & 1.81542E-08 \\
\hline & & Colorectal cancer (CRC) & 299 & -0.1683 & 70445 & 0.00351403 & 0.019444 \\
\hline & & $\begin{array}{l}\text { Head and neck squamous cell } \\
\text { carcinoma (HNSC) }\end{array}$ & 428 & -0.13371 & 124582 & 0.00559585 & 0.0178082 \\
\hline & & Lung adenocarcinoma (LUAD) & 441 & -0.12502 & 108048 & 0.00858301 & 0.0310865 \\
\hline & & Papillary thyroid carcinoma (PTC) & 557 & -0.26928 & 30480 & $1.04246 \mathrm{E}-10$ & 1.35264E-09 \\
\hline & & $\begin{array}{l}\text { Uterine corpus endometrial } \\
\text { carcinoma (UCEC) }\end{array}$ & 161 & -0.32028 & 23449 & $3.44386 \mathrm{E}-05$ & 0.000576509 \\
\hline \multirow[t]{10}{*}{ hsa-miR-20a } & \multirow[t]{10}{*}{ KAT2B } & Bladder urothelial cancer (BLCA) & 229 & -0.31374 & 25136 & $1.27 \mathrm{E}-06$ & $1.98 \mathrm{E}-05$ \\
\hline & & Breast cancer (BRCA) & 748 & -0.27776 & 25792 & $1.02 \mathrm{E}-14$ & $1.56 \mathrm{E}-13$ \\
\hline & & Colorectal cancer (CRC) & 299 & -0.37313 & 6006 & $2.61 \mathrm{E}-11$ & 1.69E-09 \\
\hline & & $\begin{array}{l}\text { Head and neck squamous cell } \\
\text { carcinoma (HNSC) }\end{array}$ & 428 & -0.37751 & 4310 & $6.05 \mathrm{E}-16$ & $5.56 \mathrm{E}-14$ \\
\hline & & Acute myeloid leukemia (LAML) & 172 & -0.2645 & 28945 & 0.000454575 & 0.00572662 \\
\hline & & Lung adenocarcinoma (LUAD) & 441 & -0.30742 & 8650 & $4.17 \mathrm{E}-11$ & $1.89 \mathrm{E}-09$ \\
\hline & & Lung squamous cell carcinoma (LUSC) & 362 & -0.31728 & 23452 & $6.57 \mathrm{E}-10$ & $1.11 \mathrm{E}-08$ \\
\hline & & Ovarian serous cystadenocarcinoma (OV) & 265 & -0.12206 & 86631 & 0.0471435 & 0.208096 \\
\hline & & Papillary thyroid carcinoma (PTC) & 557 & -0.12636 & 133650 & 0.00281234 & 0.00832219 \\
\hline & & $\begin{array}{l}\text { Uterine corpus endometrial } \\
\text { carcinoma (UCEC) }\end{array}$ & 161 & -0.21163 & 70910 & 0.00703863 & 0.0389642 \\
\hline
\end{tabular}

FDR, false discovery rate. 
ential expression profiles were analyzed, and ROC curve analyses were performed to assess the predictive power of these miRNAs and mRNAs. We found that 8 miRNAs (miR-106a, miR-15a, miR-30a, miR-30b, miR-20a, miR-20b, miR-30d and miR-30e) and 8 mRNAs (AXIN2, ITGA3, TP53INP1, TP53INP2, BCL2, PTEN, FOS and KAT2B) had higher predictive powers, and the AUC values were $>0.90$. These results indicated that these miRNAs and mRNAs are good biomarker candidates for the clinical diagnosis of PTC.

Currently, FNA is the most accurate diagnostic method used for detecting TC (38); however, up to $30 \%$ of fine-needle aspiration biopsy cytological samples are reported as 'suspicious' or 'indeterminate' (39). Therefore, additional methods to increase the sensitivity and specificity of diagnosis are highly desirable. Molecular markers, such as B-raf proto-oncogene, serine/threonine kinase (BRAF), RAS, RET/PTC, paired box 8 (PAX8)/peroxisome proliferator-activated receptor c (PPARc) or galectin-3 may be considered for determining cytology, according to the American Thyroid Association guidelines (40). In a previous study of ours, TP53INP1, TP53INP2, AXIN2 and ITGA3 were found to be differentially expressed in PTC tissues when compared with the normal tissues (34). In the present study, we revealed that aside from those 4 genes, BCL2, PTEN, FOS and KAT2B also have potential value for the diagnosis of PTC. Usually, an AUC value $>0.5$ is considered suitable for clinical diagnosis. To increase the sensitivity and specificity and the AUC, we combined the mRNA expression of AXIN2 and miR-15a in 28 patients using logistic regression analysis. The results revealed that the combination of AXIN2 and miR-15a increased diagnostic accuracy, as compared with the use of a single molecule (the sensitivity was $78.5 \%$ and the specificity was $93.7 \%$ ). These results suggest that the combination of AXIN2 and miR-15a is a strong and independent predictor for the diagnosis of PTC.

In conclusion, our data demonstrate that the combined use of miRNAs and their target mRNAs may provide a novel predicting tool for the diagnosis of PTC. The combination of miRNAs and mRNAs significantly improved the diagnostic accuracy. The data of the present study may serve as the basis for further studies on PTC diagnosis. Further studies are required to examine the mechanisms of action of different miRNAs and mRNAs in PTC.

\section{Acknowledgements}

The present study was supported by NSFC grants (30770649 and 30970682), the Research Fund for the Doctoral Program of Higher Education of China (20100061110070) and the Program for New Century Excellent Talents in University.

\section{References}

1. Siegel R, Ma J, Zou Z and Jemal A: Cancer Statistics, 2014. CA Cancer J Clin 64: 9-29, 2014.

2 Goldfarb $M$ and Casillas J: Unmet information and support needs in newly diagnosed thyroid cancer: comparison of adolescents/young adults (AYA) and older patients. J Cancer Surviv 8: 394-401, 2014.

3. Olaleye O, Ekrikpo U, Moorthy R, Lyne O, Wiseberg J, Black M and Mitchell D: Increasing incidence of differentiated thyroid cancer in South East England: 1987-2006. Eur Arch Otorhinolaryngol 268: 899-906, 2011.
4. Chen AY, Jemal A and Ward EM: Increasing incidence of differentiated thyroid cancer in the United States, 1988-2005. Cancer 115: 3801-3807, 2009.

5. Li X, Abdel-Mageed AB, Mondal D and Kandil E: MicroRNA expression profiles in differentiated thyroid cancer, a review. Int J Clin Exp Med 6: 74-80, 2013.

6. Sethi K, Sarkar S, Das S, Mohanty B and Mandal M: Biomarkers for the diagnosis of thyroid cancer. J Exp Ther Oncol 8: 341-352, 2010.

7. Torréns JI and Burch HB: Serum thyroglobulin measurement. Utility in clinical practice. Endocrinol Metab Clin North Am 30: 429-467, 2001.

8. Kebebew E and Reiff E: Patients with differentiated thyroid cancer have a venous gradient in thyroglobulin levels. Cancer 109: 1078-1081, 2007.

9. Weinberger PM, Adam BL, Gourin CG, Moretz WH III, Bollag RJ, Wang BY, Liu Z, Lee JR and Terris DJ: Association of nuclear, cytoplasmic expression of galectin-3 with beta-catenin/Wnt-pathway activation in thyroid carcinoma. Arch Otolaryngol Head Neck Surg 133: 503-510, 2007.

10. Ito Y, Yoshida H, Tomoda C, Miya A, Kobayashi K, Matsuzuka F, Kakudo K, Kuma K and Miyauchi A: HBME-1 expression in follicular tumor of the thyroid: an investigation of whether it can be used as a marker to diagnose follicular carcinoma. Anticancer Res 25: 179-182, 2005.

11. Yanaihara N, Caplen N, Bowman E, Seike M, Kumamoto K, Yi M, Stephens RM, Okamoto A, Yokota J, Tanaka T, et al: Unique microRNA molecular profiles in lung cancer diagnosis and prognosis. Cancer Cell 9: 189-198, 2006.

12. Sassen S, Miska EA and Caldas C: MicroRNA: implications for cancer. Virchows Archiv 452: 1-10, 2008.

13. Bartel DP: MicroRNAs: genomics, biogenesis, mechanism, and function. Cell 116: 281-297, 2004.

14. Baranwal S and Alahari SK: miRNA control of tumor cell invasion and metastasis. Int J Cancer 126: 1283-1290, 2010.

15. Rathod SS, Rani SB, Khan M, Muzumdar D and Shiras A: Tumor suppressive miRNA-34a suppresses cell proliferation and tumor growth of glioma stem cells by targeting Akt and Wnt signaling pathways. FEBS Open Bio 4: 485-495, 2014.

16. Pencheva $\mathrm{N}$ and Tavazoie SF: Control of metastatic progression by microRNA regulatory networks. Nat Cell Biol 15: 546-554, 2013.

17. Ell B, Qiu Q, Wei Y, Mercatali L, Ibrahim T, Amadori D and Kang Y: The microRNA-23b/27b/24 cluster promotes breast cancer lung metastasis by targeting metastasis-suppressive gene prosaposin. J Biol Chem 289: 21888-21895, 2014.

18. Gu Y, Cheng Y, Song Y, Zhang Z, Deng M, Wang C, Zheng G and He Z: MicroRNA-493 suppresses tumor growth, invasion and metastasis of lung cancer by regulating E2F1. PLoS One 9: e102602, 2014

19. Skinner HD, Lee JH, Bhutani MS, Weston B, Hofstetter W, Komaki R, Shiozaki H, Wadhwa R, Sudo K, Elimova E et al: A validated miRNA profile predicts response to therapy in esophageal adenocarcinoma. Cancer 120: 3635-3641, 2014.

20. Tumilson CA, Lea RW, Alder JE and Shaw L: Circulating microRNA biomarkers for glioma and predicting response to therapy. Mol Neurobiol 50: 545-558, 2014.

21. Saito Y, Suzuki H, Imaeda H, Matsuzaki J, Hirata K, Tsugawa H, Hibino S, Kanai Y, Saito H and Hibi T: The tumor suppressor microRNA-29c is downregulated and restored by celecoxib in human gastric cancer cells. Int J Cancer 132: 1751-1760, 2013.

22. Wang J, Zhang J, Wu J, Luo D, Su K, Shi W, Liu J, Tian Y and Wei L: MicroRNA-610 inhibits the migration and invasion of gastric cancer cells by suppressing the expression of vasodilatorstimulated phosphoprotein. Eur J Cancer 48: 1904-1913, 2012.

23. Oh HK, Tan AL, Das K, Ooi CH, Deng NT, Tan IB, Beillard E, Lee J, Ramnarayanan K, Rha SY, et al: Genomic loss of miR-486 regulates tumor progression and the OLFM4 antiapoptotic factor in gastric cancer. Clin Cancer Res 17: 2657-2667, 2011.

24. Yu S, Liu Y, Wang J, Guo Z, Zhang Q, Yu F, Zhang Y, Huang K, Li Y, Song E, et al: Circulating microRNA profiles as potential biomarkers for diagnosis of papillary thyroid carcinoma. J Clin Endocrinol Metab 97: 2084-2092, 2012.

25. Etheridge A, Lee I, Hood L, Galas D and Wang K: Extracellular microRNA: a new source of biomarkers. Mutat Res 717: 85-90, 2011.

26. Cortez MA, Welsh JW and Calin GA: Circulating microRNAs as noninvasive biomarkers in breast cancer. Recent Results Cancer Res 195: 151-161, 2012. 
27. Yip L, Kelly L, Shuai Y, Armstrong MJ, Nikiforov YE, Carty SE and Nikiforova MN: MicroRNA signature distinguishes the degree of aggressiveness of papillary thyroid carcinoma. Ann Surg Oncol 18: 2035-2041, 2011.

28. Fassina A, Cappellesso R, Simonato F, Siri M, Ventura L, Tosato F, Busund LT, Pelizzo MR and Fassan M: A 4-MicroRNA signature can discriminate primary lymphomas from anaplastic carcinomas in thyroid cytology smears. Cancer Cytopathol 122: 274-281, 2014.

29. Nikiforova MN, Tseng GC, Steward D, Diorio D and Nikiforov YE: MicroRNA expression profiling of thyroid tumors: biological significance and diagnostic utility. J Clin Endocrinol Metab 93: 1600-1608, 2008.

30. Hébrant A, Dom G, Dewaele M, Andry G, Trésallet C, Leteurtre E, Dumont JE and Maenhaut C: mRNA expression in papillary and anaplastic thyroid carcinoma: molecular anatomy of a killing switch. PLoS One 7: e37807, 2012.

31. Lee JC, Zhao JT, Clifton-Bligh RJ, Gill A, Gundara JS, Ip JC Glover A, Sywak MS, Delbridge LW, Robinson BG and Sidhu SB: MicroRNA-222 and microRNA-146b are tissue and circulating biomarkers of recurrent papillary thyroid cancer. Cancer 119: 4358-4365, 2013.

32. Ruggeri RM, Campennì A, Baldari $S$, Trimarchi $F$ and Trovato $M$ : What is New on Thyroid Cancer Biomarkers. Biomark Insights 3: 237-252, 2008

33. He M, Zhao Y, Yi H, Sun H, Liu X and Ma S: The combination of TP53INP1, TP53INP2 and AXIN2: potential biomarkers in papillary thyroid carcinoma. Endocrine 48: 712-720, 2015.

34. Liu X, He M, Hou Y, Liang B, Zhao L, Ma S, Yu Y and Liu X Expression profiles of microRNAs and their target genes in papillary thyroid carcinoma. Oncol Rep 29: 1415-1420, 2013.
35. He H, Jazdzewski K, Li W, Liyanarachchi S, Nagy R, Volinia S, Calin GA, Liu CG, Franssila K, Suster S, et al: The role of microRNA genes in papillary thyroid carcinoma. Proc Natl Acad Sci USA 102: 19075-19080, 2005.

36. Pallante P, Visone R, Ferracin M, Ferraro A, Berlingieri MT, Troncone G, Chiappetta G, Liu CG, Santoro M, Negrini M, et al: MicroRNA deregulation in human thyroid papillary carcinomas. Endocr Relat Cancer 13: 497-508, 2006.

37. Shen R, Liyanarachchi S, Li W, Wakely PE Jr, Saji M, Huang J, Nagy R, Farrell T, Ringel MD, de la Chapelle A, et al: MicroRNA signature in thyroid fine needle aspiration cytology applied to 'atypia of undetermined significance' cases. Thyroid 22: 9-16, 2012.

38. Gharib $\mathrm{H}$ : Changing trends in thyroid practice: understanding nodular thyroid disease. Endocr Pract 10: 31-39, 2004

39. Prasad NB, Somervell H, Tufano RP, Dackiw AP, Marohn MR, Califano JA, Wang Y, Westra WH, Clark DP, Umbricht CB, et al: Identification of genes differentially expressed in benign versus malignant thyroid tumors. Clin Cancer Res 14: 3327-3337, 2008.

40. American Thyroid Association (ATA) Guidelines Taskforce on Thyroid Nodules and Differentiated Thyroid Cancer, Cooper DS, Doherty GM, Haugen BR, Kloos RT, Lee SL, Mandel SJ, Mazzaferri EL, McIver B, Pacini F, Schlumberger M, et al: Revised American Thyroid Association management guidelines for patients with thyroid nodules and differentiated thyroid cancer. Thyroid 19: 1167-1214, 2009. 